PART V

\title{
GROUND BASED AND EXTRATERRESTRIAL OBSERVATIONS OF STELLAR FLUX
}




\title{
GROUND BASED AND
}

\section{EXTRATERRESTRIAL OBSERVATIONS OF STELLAR FLUX}

\author{
A. D. CODE \\ Washburn Observatory, University of Wisconsin, Madison, Wis., U.S.A.
}

\begin{abstract}
The significance of recent improvements made in the absolute monochromatic flux measurements of the Sun and Vega are discussed with a special emphasis on the absolute calibration of the $V$-magnitude of stars. The difficulties relating to the determination of vacuum ultra-violet fluxes are outlined and the improvements that can be achieved using synchroton radiation as the fundamental laboratory source are presented. Colour indices derived from OAO-2 observation are given for about 80 early type stars and the $(1700-V)$ colour is discussed in terms of interstellar reddening and sensitivity to effective temperature. Basic problems requiring further investigation are finally outlined.
\end{abstract}

\section{Introduction}

About fifteen years ago I prepared a review of the then current status of observations of stellar flux. I would like to describe here some of the significant advances that have occurred since that time and conclude with several of the problems which still require attention.

The advances made in laboratory spectroradiometry have been important in improving our knowledge of stellar radiation. These include the more precise determination of the thermodynamic temperature scale and the establishment of spectral irradiance standards at the National Bureau of Standards in the United States and at Heidelberg, for example. Significant advances in instrumentation and development of reliable portable radiation sources such as the Copper black body furnace have resulted in improvement in accuracy. The careful measurements of Hayes and of Oke and Schild have taken advantage of these techniques for measurements in the visual region for stars. Our knowledge of the solar flux has been considerably improved by the ability to perform measurements from altitudes where variable components, such as water vapor and aerosols, are reduced to an insignificant level.

The extension of stellar measurements to the infrared and vacuum ultraviolet has opened up the opportunity to determine empirical bolometric corrections while substantially increasing our understanding of the stellar structure and of the interstellar and circumstellar medium. The intensity interferometer measurements of stellar angular diameters by Hanbury-Brown and associates has made possible the determination of the true emergent flux from these objects.

Until recently the measurement of absolute fluxes in the vacuum ultraviolet has been particularly difficult because of the lack of suitable radiation sources. The application of synchrotron radiation from a synchrotron storage ring has represented a major advance in vacuum ultraviolet energy calibration.

These advances in observational astronomy have been accompanied by equally impressive achievements on the theoretical side. The utilization of large digital computers 
has made possible the calculation of relatively sophisticated model atmospheres. Grids of models exist which include line blanketing from many individual lines as well as statistical line blanketing. In addition the effects of NLTE and of extended atmospheres have been investigated.

These advancements have clarified many features but also presented substantial new problems and some significant disagreements.

In what follows I shall describe some of the above investigations in more detail.

\section{Solar Spectrum}

Although the Sun has been studied extensively, observations of the Sun as a star have been limited. Until recently the solar flux was probably not as well determined as that of Vega and the best data on the sun were found using the monochromatic intensities at the center of the disk and the measured limb darkening. Furthermore, the determination of $U, B, V$ magnitudes and MK spectral type are complicated by the inability to use the same instrumentation over this dynamic range of some 27 mag.

The most frequently employed determinations of the solar flux have been the compilation of Johnson (1954) and that of Nicolet (1951) derived using limb darkening data. More recently Labs and Neckel (1968) obtained measurements of the central intensity from 3300-12500 $\AA$ from the Jungfraujoch Scientific Station, Switzerland (altitude $3.6 \mathrm{~km}$ ) which were combined with center-limb variations and line blanketing coefficients to obtain a solar flux curve. All these studies include inaccuracies in the evaluation of the atmospheric attenuation and the inaccuracies in determining centerlimb variations and the line blanketing coefficients.

Most compilations of the solar spectrum below $3330 \AA$ have been based on NRL rocket measurements given by Tousey (1963) (cf. Furukawa et al., 1967). For the purpose of determining the total integrated solar flux or effective temperature, this data is completely satisfactory since the total flux shortward of $3330 \AA$ is less than $3 \%$ of the total radiation. In studies of the ultraviolet albedo of planets, however, it is clear that these complications of the solar UV spectrum contain systematic errors (cf. Wallace et al., 1972) and that the extreme UV is variable. Recently Broadfoot (1972) has measured the solar flux between $2000-3000 \AA$ with an aerobee rocket spectrometer and found good agreement with the earlier data except in the region of $2200 \AA$ where the previous spectra have been depressed.

For the infrared between 8.6 and $13 \mu$ the determinations by Saidy (1960) appear satisfactory. This region of the spectrum contains only $0.1 \%$ of the total energy and hence is primarily of importance for spectral investigation in this region.

Recently the solar flux from $3000 \AA$ to $25000 \AA$ has been carefully determined by direct high resolution measurements from a NASA Convair 990 aircraft (Arvesen et al., 1969). Eleven long duration flights between 11.5 and $12.5 \mathrm{~km}$ have been carried out using a particularly carefully designed instrumental technique. The results are independent of any assumptions about line blanketing or limb-darkening and free of atmospheric transmission variations due to aerosols or water vapor. The total flux 
value is considered good to $3 \%$, while the monochromatic fluxes become uncertain to about $6 \%$ at $3200 \AA, 7 \%$ at $3100 \AA$, and $25 \%$ at $3000 \AA$, the main source of uncertainty being due to the calibration of the standard lamp. The agreement with Labs and Neckel is good.

On the basis of existing measurements it would appear that the most reliable solar spectrum from 2000-3000 $\AA$ is the Broadfoot (1972) spectrum and from 3100-25000 $\AA$ the Arvesen (1969) spectrum, while longward of $25000 \AA$ a $5800 \mathrm{~K}$ grey body approximation joined to the Saidy (1960) spectrum should give an adequate representation of the solar continuum.

The total integrated flux of the Sun resulting from this spectrum is $1360 \mathrm{~W} \mathrm{~m}^{-2}$ (Duncan, 1969) which corresponds to an effective temperature for the Sun of $5770 \mathrm{~K}$. It is more difficult to relate these results to measurements of stellar flux. The spectral type of G2 V for the Sun that has been determined by Morgan (Stebbins and Kron, 1957) is as well determined as that for other stars. However, the determination of the magnitude and color of the Sun has presented difficulties.

The determination of the visual magnitude and color of the sun by Stebbins and Kron (1957) was one of the most extensive direct comparisons of the Sun and stars. They obtained a visual magnitude $V=-26.73 \pm 0.03$ and a $B-V=+0.63$. Martinov (1959) rediscussed all data on the visual magnitude of the Sun and obtained a value of $V=-26.80 \pm 0.3$, while Johnson (1965) reviewed all recent data obtained by various indirect means along with the Stebbins and Kron measurements and arrived at a value of $V=-26.74$. Determinations of the $B-V$ color since Stebbins and Kron by indirect means have ranged from +0.65 to +0.68 and the most recent determination by Fernie et al. (1971) yields $(B-V)=+0.628$.

The Sun as a star is probably closely represented by a spectral type $\mathrm{G} 2 \mathrm{~V}$, a $B-V$ color of +0.63 , and visual magnitude of $V=-26.74$. We shall return to this comparison, keeping in mind the variations described above after reviewing the status of stellar flux determinations.

\section{Stellar Spectra}

The energy distribution of Vega adopted by the author (Code, 1960) was a composite one including observations made at Jungfraujoch, by Kienle and his co-workers, Williams and Hall, as well as observations by Whitford and Code in the red. Subsequent observations by Bahner (1963) and others indicated that the Balmer jump was too small by about $0 \mathrm{~m} \cdot 13$. Furthermore the Paschen continuum could not be satisfactorily represented by model atmosphere calculations. Oke (1964) derived an energy curve for Vega based upon the best model representation known in 1964. Investigations by Gluschneve (1964), Kharitonov (1963), and Willstrop (1965) provided improved calibrations although discrepancies as great as $10 \%$ persisted.

A new and careful spectrophotometric calibration by Hayes (1967) resulted in a continuous energy distribution that was in good agreement with the predictions of model atmospheres and incidentally with the early work of Kienle. Oke and Schild (1970) carried out a program designed to measure the absolute monochromatic flux 
of Vega from $3300 \AA$ to $10000 \AA$ utilizing a telescope, scanner and photometric system specifically constructed for these observations. Three radiation sources - a tungsten ribbon lamp, a copper-point black body and a platinum-point black body - were directly compared with Vega. In the spectral interval from 4000-6000 $\AA$ they quote errors of $\pm 2 \%$. Beyond this range in the infrared and ultraviolet, scatter as high as $6 \%$ was found, but no systematic differences between different sets of data. In the region from $4000-6000 \AA$ the agreement with Hayes is excellent. Figure 1 compares the

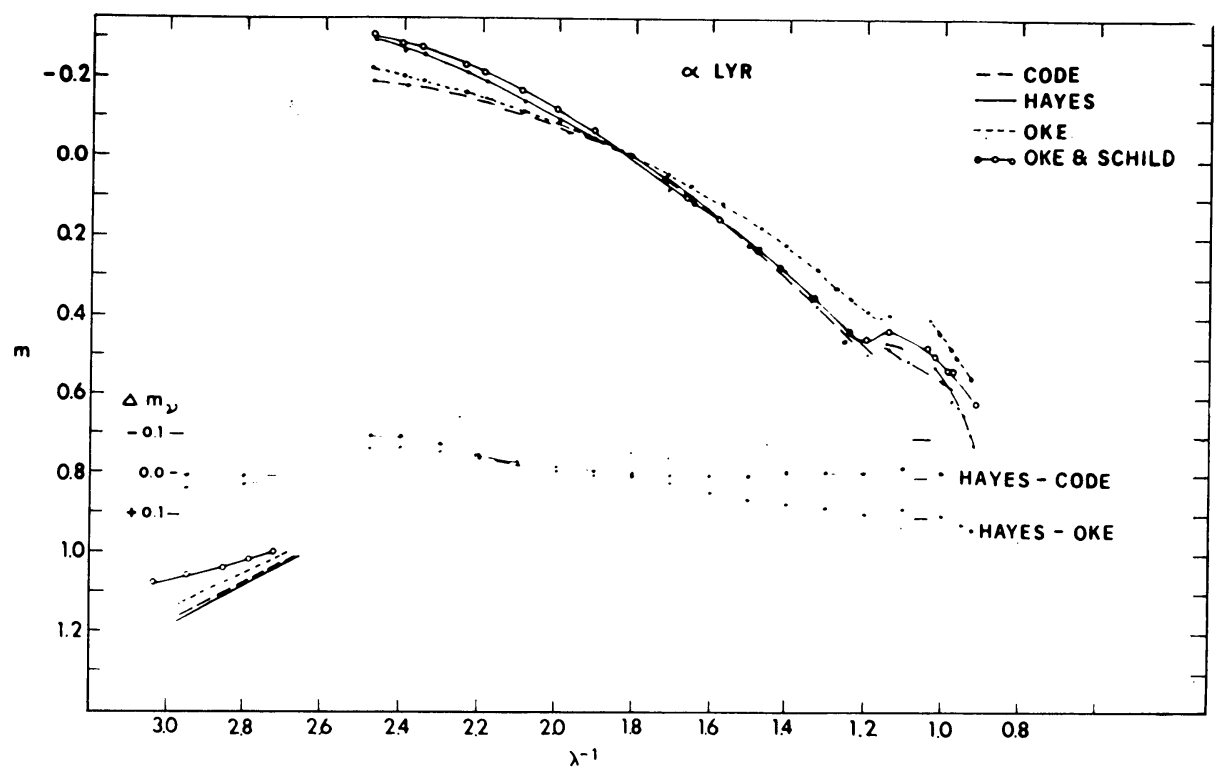

Fig. 1. Comparison of different calibrations for Vega.

results of this investigation with several of the earlier energy curves of Vega. There is still a disquieting discrepancy shortward of the Balmer discontinuity and longward of the Paschen jump. I believe that these differences represent a realistic estimate of the overall uncertainties in the energy distribution of Vega and can suggest nothing better at the present time than to adopt the mean of Hayes (1970) and Oke and Schild (1970) as the best representation of the monochromatic flux of Vega in the spectral interval 3300 to $10000 \AA$.

On the basis of their investigation Oke and Schild find the absolute monochromatic flux at $5556 \AA$ for $\alpha$ Lyr to be $3.36 \times 10^{-9} \mathrm{ergs} \mathrm{s}^{-1} \mathrm{~cm}^{-2} \AA^{-1}$. Adopting an effective wavelength of $5480 \AA$ for the $V$-filter of the $U B V$ system they find a value of $3.64 \times$ $\times 10^{-9} \mathrm{ergs} \mathrm{s}^{-1} \mathrm{~cm}^{-2} \AA^{-1}$ for a star of visual magnitude $V=0.00$. This number is an important quantity for providing a means of calibrating the $V$-magnitude of a star. The precise meaning of an energy curve derived from a wide band filter photometer is of course dependent upon the detailed energy distribution over the filter band pass and hence ambiguous. I shall, however, adopt the point of view that the $V$-magnitude of a star represents the integral effective intensity (Code, 1960) determined by a photometer 
with a band pass characteristic as tabulated for zero air mass by Matthews and Sandage (1963). This sensitivity function was derived by the author based on Johnson's (1955) tabulated filter and 1P21 response and the reflectivity of two aluminum mirrors. If one integrates over this band pass the Oke-Schild energy curve of Vega, the integral effective intensity corresponds to $3.58 \times 10^{-9} \mathrm{ergs} \mathrm{s}^{-1} \mathrm{~cm}^{-2} \AA^{-1}$. It is of interest to compare this result with that found by integrating the Arvesen solar spectrum over the $V$ band pass. If we adopt a visual magnitude for the Sun of $V_{\odot}=-26.74$ we find an integral effective intensity of $3.65 \times 10^{-9} \mathrm{ergs} \mathrm{s}^{-1} \mathrm{~cm}^{-2} \AA^{-1}$, while the range of values for the visual magnitude of the Sun yield values from 3.68 to 3.45. A mean value for the integral effective intensity of a star of visual magnitude $V=0.00$ as determined from the solar spectrum and from Vega is $3.61 \pm 0.10 \times 10^{-9} \mathrm{ergs} \mathrm{s}^{-1} \mathrm{~cm}^{-2}$ $\AA^{-1}$. This result is not particularly sensitive to the detailed shape of the $V$ filter sensitivity curve and within the uncertainty quoted is independent of any color term for solar type stars or earlier.

That there are no large systematic differences between the energy determinations of stars and of the Sun is shown in Figure 2, where the difference in magnitudes between

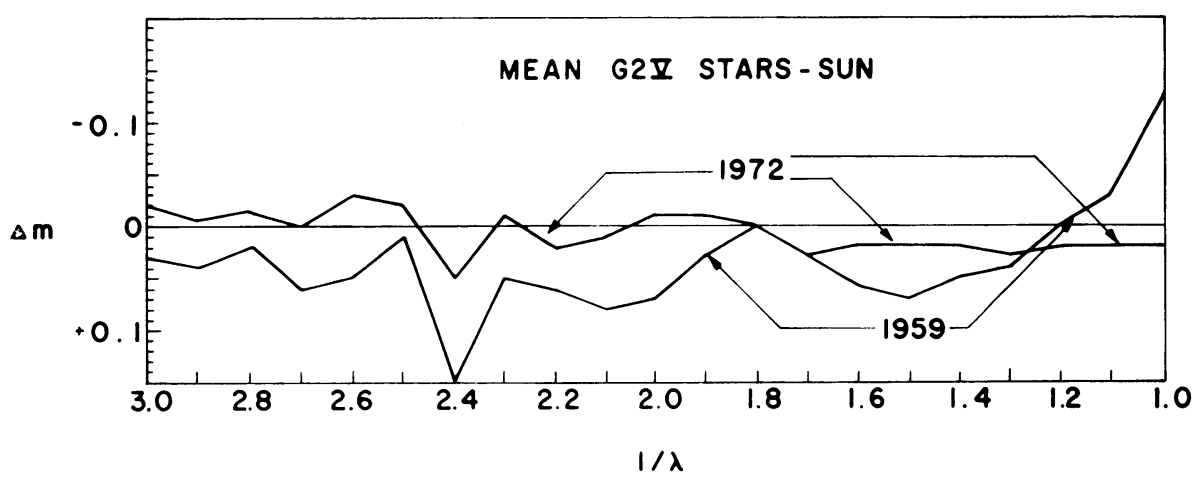

Fig. 2. Differences between the energy determinations of stars and of the Sun.

the mean energy distribution of solar type stars whose average $B-V$ color is \pm 0.63 and of the Sun are compared. The 1959 curve was determined by the author (Code, 1960) based on earlier data. The 1972 curve is for the same stars using the mean of Hayes and the Oke-Schild calibration of Vega and the Arvesen et al. solar spectrum. The curves are normalized at $5556 \AA$ and show remarkable agreement over the entire spectral region.

The determination of the ultraviolet energy distribution of stars is still in a relatively primitive state, particularly shortward of $1500 \AA$. Where we speak of differences of a few percent in the visual, discrepancies of a factor of 3 or 4 exist in the far UV. One of the basic difficulties has been the lack of a satisfactory fundamental radiation source. The intensity of a tungsten lamp or carbon arc falls off too rapidly to be of use below $2500 \AA$. A black body source must be operated at temperatures well above the melting point of any metals and hence the source must be in a plasma state. Boldt has 
succeeded in developing an optically thick arc running at a temperature of about $14000 \mathrm{~K}$ which in principle can yield calibrations to about $20 \%$ at $1000 \AA$.

Most energy measurements of stars in the ultraviolet have depended upon a more indirect calibration. In general a laboratory reference photomultiplier with a sodium salicylate coating has been calibrated longward of $2500 \AA$ and the quantum efficiency of the sodium salicylate has been assumed to be uniform as a function of wavelength. This assumption has usually been checked by the use of nitric oxide ion chambers at $1216 \AA$. A review of methods of intensity calibration has been given by McWhirter (1971). Descriptions of the calibration of specific payloads are usually presented along with UV data (cf. Carruthers, 1969; Stuart, 1969; Evans, 1972). While accuracies of 15 to $30 \%$ are sometimes quoted, differences up to a factor of 5 exist. The history of ultraviolet spectrophotometry has been one of the large flux deficiencies relative to theoretical predictions, which have moved to shorter and shorter wavelengths as techniques have improved. It is now generally agreed that the observed fluxes are in reasonable agreement with theory longward of $2000 \AA$, while evidence is accumulating that there are no large flux deficiencies in the 1200-1500 $\AA$ region (Bless and Code, 1972).

One of the most promising fundamental sources of ultra-violet radiation is the synchrotron radiation from high energy electrons circulating in a storage ring. The University of Wisconsin operates a facility consisting of a synchrotron which accelerates electrons to about $50 \mathrm{MeV}$, injects them into a storage ring in which, after further acceleration to about $240 \mathrm{MeV}$, they can circulate for many hours. The source is stable in time, has a continuous energy distribution similar to a B5 star and can be calibrated absolutely. The fact that the energy distribution is similar to an early type star removes one serious source or error that has plagued ultraviolet calibrations in the past, namely scattered light problems and low rapidly changing intensities. The absolute intensity of the beam can be determined independently of any thermodynamic temperature scales or previous absolute radiation standards. This comes about because it is possible to measure the radiation from a single electron and use theory to determine the energy distribution, which is very insensitive to the electron energy longward of $1000 \AA$ for $240 \mathrm{MeV}$ electrons. We may check the theoretical calculations by measuring the angular distribution and polarization of the radiation.

We usually start with about 50 electrons and measure the step-wise decrease in intensity of the radiation each time an electron is ejected from the beam and thus one can determine the number of electrons producing a given signal. We have calibrated rocket payloads directly in the essentially collimated synchrotron radiation both before and after flight (Gaide, 1971; Bless et al., 1972). The calibration obtained by this method agrees well with our OAO-2 preflight calibration and is the basis of our present absolute energy determinations. Figure 3 shows the energy distribution of $\eta$ UMa as measured by OAO- 2 employing this calibration. The solid curve is from OAO-2 spectral scans. The solid circles are the results of filter measurements obtained with a rocket calibration payload. The open circles are the results of Schild et al. (1971), while the dotted curve results from the Hayes calibration. The circled 
cross is an OAO-2 filter measurement derived from solar type stars and the Arvesen solar spectrum. The energy distribution is similar to that of a blanketed model atmosphere with an effective temperature of the order of $17000 \mathrm{~K}$. Evans (1972) has carried out a continuing program on absolute calibration in the ultraviolet which diverges from our results substantially shortward of $2000 \AA$. The Evans calibration yields an energy curve for $\eta$ UMa shown by the dash-dot curve in Figure 3. The results of Moos et al. are shown by the dashed curve. The Evans calibration has been an

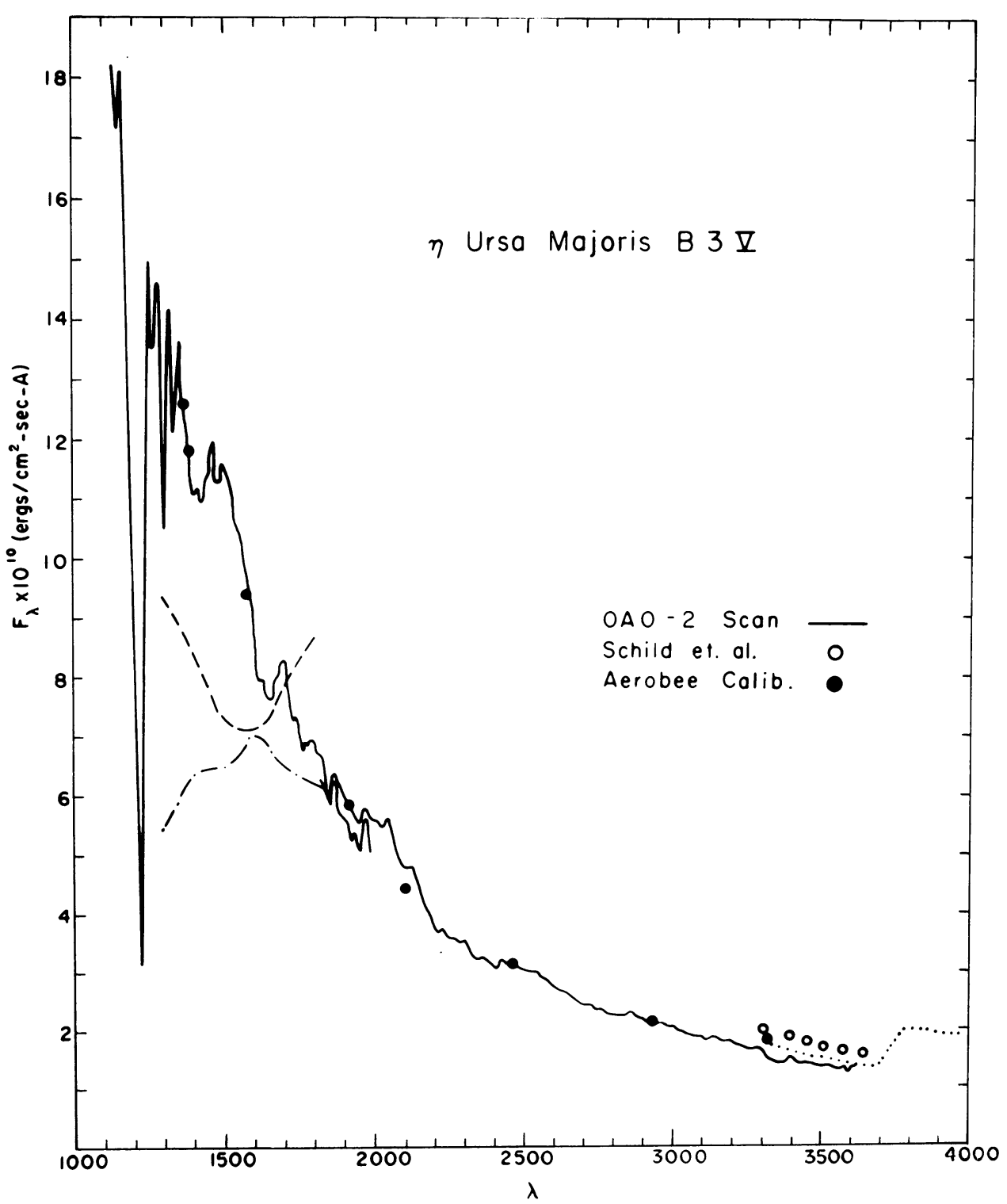

Fig. 3. The energy distribution of $\eta \mathrm{UMa}$ as measured by OAO-2 employing this calibration. 
extensive and careful one and the source of the discrepancy is at present unknown. The results of Carruthers are in agreement with the Wisconsin determinations and the results of Stuart yield somewhat larger fluxes than the Wisconsin results. The ratio of total integrated flux for $\eta$ UMa implied by the difference between the Wisconsin and Goddard calibration is 2 which would indicate an effective temperature of the order of $15000 \mathrm{~K}$ for $\eta \mathrm{UMa}$ for the Goddard result.

Figure 4 shows the energy distribution of $\mathrm{S}$ Mon based on the Wisconsin calibration. The filled circles are the Geneva balloon results (Navach, 1972). These observations have been corrected for an interstellar color excess of $E_{B-V}=0.07$ using the

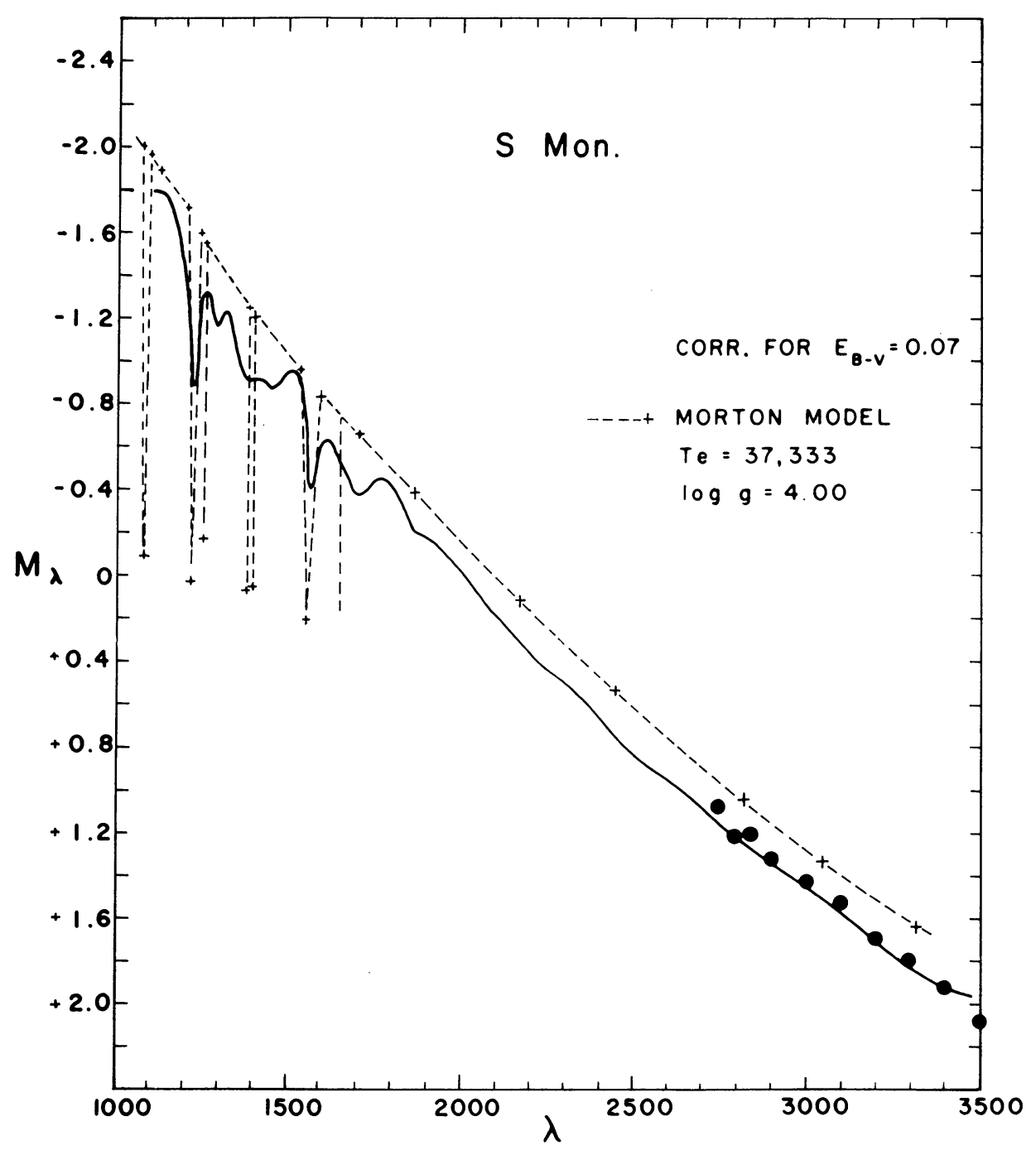

Fig. 4. Energy distribution of $\mathrm{S}$ Mon based on the Wisconsin calibration. 


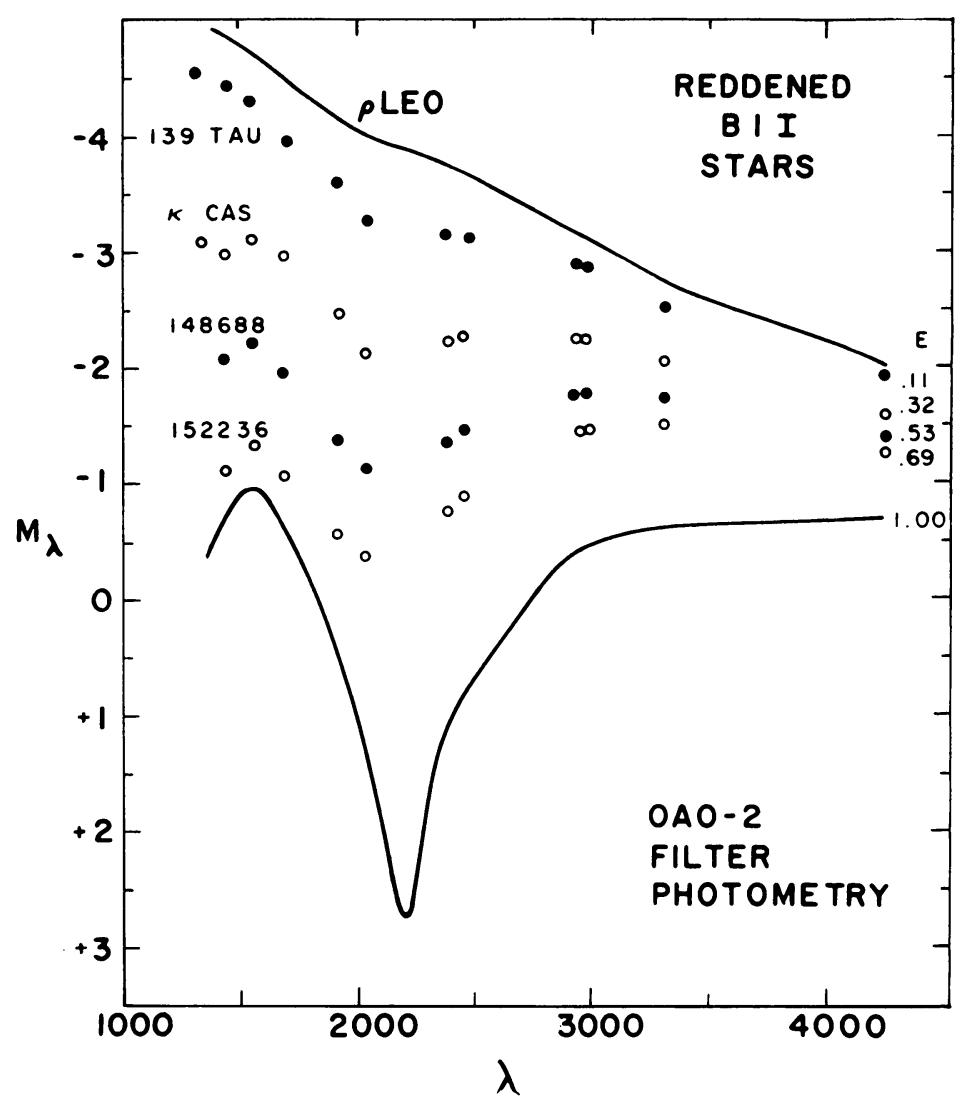

Fig. 5. Energy distribution of a number of reddened B1 I stars from OAO filter observations

mean extinction curve of Bless and Savage (1972) and compared with a Morton blanketed model for $T_{\text {eff }}=37333 \mathrm{~K}$.

The Wisconsin absolute calibration has been well determined longward of $1300 \AA$ but is not based on a fundamental calibration shortward of $1300 \AA$, although it agrees well with the Lyman alpha calibration by Blamont of his OGO-V instrument. It is clear, however, that much work remains to be done on the energy calibration shortward of $2000 \AA$.

\section{Interstellar Extinction}

The comparison of ultraviolet measurements of stars with theoretical predictions is limited by our knowledge of the interstellar extinction curve. The discussion by Bless and Savage (1972) shows that the extinction is large, variable and non-linear in the UV. A $(B-V)$ color excess of only $0^{\mathrm{m}} \cdot 1$ could imply extinction at $1250 \AA$ of as little as $0 \mathrm{~m}$. 3 or as much as 0.7 . They have found, however, that stars of similar $B-V$ colors and spectral types do have the same spectral distribution in the ultraviolet and therefore the suggestion by Underhill (1972), that variations in reddening are due to line 
blocking, is not verified. Figure 5 shows the energy distribution of a number of reddened B1 I stars from OAO filter observations along with the computed curve for a $B-V$ color excess of $1 \mathrm{mag}$. The agreement for these stars with the mean extinction curve of Bless and Savage is good.

Figure 6 shows a spectral scan of $\eta$ UMa. The internal accuracy is very good (within $2 \%$ ). The region in the neighborhood of $1700 \AA$ shows a pronounced maximum. We have measured monochromatic colors at $1700 \AA$ for many early type stars. Table I contains the results for 65 little reddened stars. The mean ratio of $E_{1700-V} / E_{B-V}$ is 4.4 .

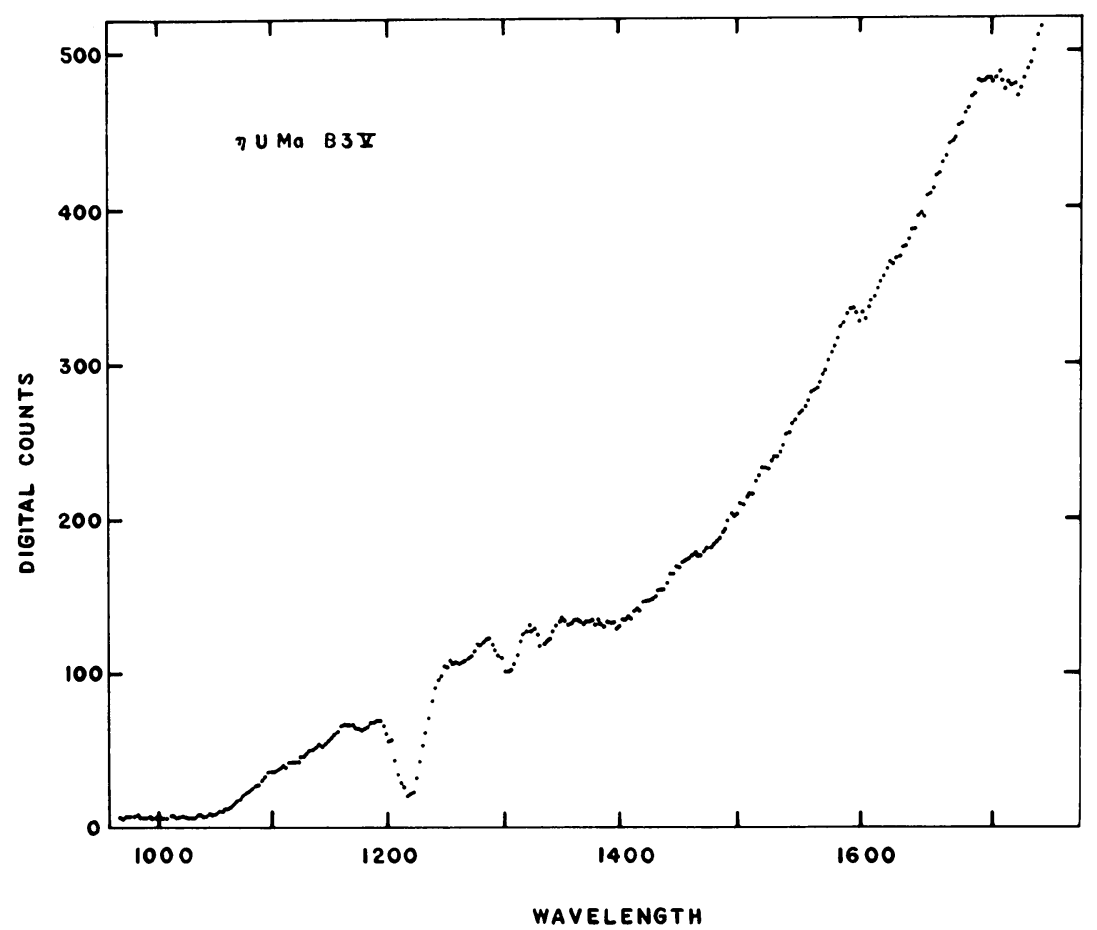

Fig. 6. Spectral scan of $\eta \mathrm{U} \mathrm{Ma}$.

I have determined $1700-V$ normal colors for these stars. A two color plot employing these data may be found in the paper of Bless and Savage (1972).

We have chosen $1700 \AA$ for several reasons. It is a high point in the continuum and also relatively free of predicted lines. It occurs at a minimum in the interstellar extinction curve, and finally is at a wavelength in the Balmer continuum which is least sensitive to changes in gravity in model atmosphere calculations. The $1700-V$ is of course an order of magnitude more sensitive to changes in temperature than $B-V$. The color index is based on the Wisconsin absolute calibration and agrees very well with Morton's blanketed models. Identification of the $(1700-V)_{0}$ colors with model atmospheres provides an effective temperature scale similar to those recently proposed on the basis of other criteria. 
TABLE I

$(1700-V)$ Fluxes

\begin{tabular}{|c|c|c|c|c|c|c|c|c|}
\hline HR No. & Star & Sp. Type & $V$ & $(B-V)$ & $1700-V$ & $E_{1700}$ & $(B-V)_{0}$ & $(1700-V)_{0}$ \\
\hline 2456 & S Mon & O7 & 4.66 & -0.24 & -3.84 & 0.35 & -0.32 & -4.19 \\
\hline 3165 & $\zeta$ Pup & $\mathrm{O} 5 \mathrm{f}$ & 2.25 & -0.29 & -4.00 & 0.18 & -0.33 & -4.18 \\
\hline 3207 & $\gamma$ Vel & WC8 & 1.82 & -0.27 & -3.97 & $0.22 \mathrm{P}$ & -0.32 & -4.19 \\
\hline 1228 & $\xi$ Per & 07.5 & 4.06 & +0.01 & -2.80 & $1.32_{4}^{\mathrm{K}}$ & -0.32 & -4.12 \\
\hline 1899 & $\iota$ Ori & O9 III & 2.76 & -0.23 & -3.77 & $0.35^{4.00}$ & -0.31 & -4.12 \\
\hline 8622 & $10 \mathrm{Lac}$ & $09 \mathrm{~V}$ & 4.88 & -0.20 & -3.64 & 0.48 & -0.31 & -4.12 \\
\hline 1931 & $\sigma$ Ori & $09.5 \mathrm{~V}$ & 3.83 & -0.24 & -3.89 & $0.26 \mathrm{R}$ & -0.30 & -4.15 \\
\hline 6175 & $\zeta$ Oph & $09.5 \mathrm{~V}_{\mathrm{nn}}$ & 2.57 & +0.02 & -2.51 & $1.57 \mathrm{~K}$ & -0.30 & -4.08 \\
\hline $1851-52$ & $\delta$ Ori & $\begin{array}{l}09.5 \text { II-III } \\
+ \text { B2 V }\end{array}$ & 2.20 & -0.21 & -3.58 & $0.35^{4.90}$ & -0.29 & -3.93 \\
\hline $1948-49$ & $\zeta$ Ori & $09.5 \mathrm{Ib}$ & 1.74 & -0.21 & -3.45 & $0.26 \mathrm{p}$ & -0.27 & -3.71 \\
\hline 1542 & $\alpha \mathrm{Cam}$ & $09.5 \mathrm{Ia}$ & 4.29 & +0.03 & -2.23 & $1.47_{490}^{\mathrm{R}}$ & -0.27 & -3.70 \\
\hline 1855 & $v$ Ori & B0 V & 4.63 & -0.26 & -4.07 & $0.18^{4.90}$ & -0.30 & -4.25 \\
\hline 6165 & $\tau$ Sco & B0 V & 2.82 & -0.25 & -3.93 & $0.22 \mathrm{R}$ & -0.30 & -4.15 \\
\hline 5953 & $\delta$ Sco & BO V & 2.33 & -0.10 & -3.12 & $0.98^{\mathrm{R}}$ & -0.30 & -4.10 \\
\hline 1903 & $\varepsilon$ Ori & B0 Ia & 1.70 & -0.19 & -3.22 & $0.22^{4.90}$ & -0.24 & -3.44 \\
\hline 1788 & $\eta$ Ori & $\mathrm{B} 0.5 \mathrm{~V}_{\mathrm{nn}}$ & 3.35 & -0.19 & -3.29 & 0.40 & -0.28 & -3.69 \\
\hline 1756 & $\lambda$ Lep & B0.5 IV & 4.29 & -0.25 & -3.88 & $0.13 \mathrm{R}$ & -0.28 & -4.01 \\
\hline 5984 & $\beta^{1} \mathrm{Sco}$ & $\mathrm{B} 0.5 \mathrm{~V}+\mathrm{B} 2 \mathrm{~V}$ & 2.55 & -0.08 & -2.99 & $0.70_{350}^{R}$ & -0.28 & -3.69 \\
\hline 1220 & $\varepsilon$ Per & B0.5 III & 2.89 & -0.18 & -3.34 & $0.44^{3.50}$ & -0.28 & -3.78 \\
\hline 4853 & $\beta$ Cru & B0.5 III & 1.24 & -0.24 & -3.69 & $0.18_{\mathrm{R}}$ & -0.28 & -3.87 \\
\hline 7446 & $\kappa \mathrm{Aq} 1$ & B0.5 $\mathrm{III}_{\mathrm{n}}$ & 4.95 & 0.00 & -2.51 & $1.37^{R}$ & -0.28 & -3.88 \\
\hline \multirow[t]{2}{*}{2004} & $\kappa$ Ori & B0.5 Ia & 2.06 & -0.18 & -3.23 & $0.18^{4.90}$ & -0.22 & -3.41 \\
\hline & 42 Ori & B1 V & 4.60 & -0.19 & -3.36 & 0.31 & -0.26 & -3.67 \\
\hline 1789 & 25 Ori & $\mathrm{B} 1 \mathrm{~V}_{\mathrm{n}}$ & 4.95 & -0.21 & -3.47 & 0.22 & -0.26 & -3.69 \\
\hline 5056 & $\alpha \mathrm{Vir}$ & $\mathrm{B} 1 \mathrm{~V}+\mathrm{B} 3$ & 0.96 & -0.25 & -3.54 & 0.04 & -0.26 & -3.58 \\
\hline 5944 & $\pi$ Sco & $\mathrm{B} 1 \mathrm{~V}+\mathrm{B} 2$ & 2.92 & -0.19 & -3.47 & $0.31_{R}$ & -0.26 & -3.78 \\
\hline 5993 & $\omega^{1} \mathrm{Sco}$ & B1 V & 3.99 & -0.04 & -2.89 & $0.77_{350}^{\mathrm{R}}$ & -0.26 & -3.66 \\
\hline 2571 & $15 \mathrm{CMa}$ & B1 III & 4.82 & -0.21 & -3.39 & $0.22^{3.50}$ & -0.26 & -3.61 \\
\hline 5267 & $\beta$ Cen & B1 III & 0.61 & -0.23 & -3.56 & 0.13 & -0.26 & -3.69 \\
\hline 8238 & $\beta$ Cep & B1 III & 3.20 & -0.21 & -3.40 & $0.22 \mathrm{R}$ & -0.26 & -3.62 \\
\hline 6084 & $\sigma$ Sco & B1 III & 2.89 & +0.14 & -2.33 & $1.08_{27}^{\mathrm{R}}$ & -0.26 & -3.41 \\
\hline 2294 & $\beta$ СМа & B1 II-III & 1.98 & -0.24 & -3.53 & $0.04_{\mathrm{R}}^{2.7}$ & -0.25 & -3.57 \\
\hline 1203 & $\zeta$ Per & B1 Ib & 2.86 & +0.10 & -1.79 & $1.16_{400}^{\mathrm{K}}$ & -0.19 & -2.95 \\
\hline 2084 & $139 \mathrm{Tau}$ & B1 Ib & 4.83 & -0.07 & -2.73 & $0.42_{\mathrm{R}}^{4.00}$ & -0.19 & -3.15 \\
\hline 6247 & $\mu^{1}$ Sco & B1.5 IV & 3.02 & -0.23 & -3.38 & $0.09_{350}^{R}$ & -0.25 & -3.47 \\
\hline 5695 & $\delta$ Lup & B1.5 IV & 3.21 & -0.22 & -3.46 & $0.13^{3.50}$ & -0.25 & -3.59 \\
\hline 6580 & $\kappa \mathrm{Sco}$ & B1.5 III & 2.41 & -0.20 & -3.50 & 0.22 & -0.25 & -3.72 \\
\hline 5469 & $\alpha$ Lup & B1.5 III & 2.31 & -0.21 & -3.25 & 0.18 & -0.25 & -3.43 \\
\hline 6028 & 13 Sco & B2 V & 4.58 & -0.15 & -3.10 & 0.40 & -0.24 & -3.50 \\
\hline 5708 & $\varepsilon$ Lup & B2 IV-V & 3.36 & -0.17 & -3.14 & 0.31 & -0.24 & -3.45 \\
\hline 153 & $\zeta \mathrm{Cas}$ & B2 IV & 3.66 & -0.18 & -3.17 & 0.26 & -0.24 & -3.43 \\
\hline 39 & $\gamma \mathrm{Peg}$ & B2 IV & 2.86 & -0.21 & -3.46 & 0.13 & -0.24 & -3.59 \\
\hline 5248 & $\phi$ Cent & B2 IV & 3.82 & -0.21 & -3.35 & 0.13 & -0.24 & -3.48 \\
\hline 5571 & $\beta$ Lup & B2 III & 2.67 & -0.21 & -3.46 & 0.13 & -0.24 & -3.59 \\
\hline 1790 & $\gamma$ Ori & B2 III & 1.66 & -0.21 & -3.32 & 0.13 & -0.24 & -3.45 \\
\hline 2618 & $\varepsilon \mathrm{CMa}$ & B2 II & 1.50 & -0.21 & -3.17 & 0.09 & -0.23 & -3.26 \\
\hline 5948 & $\eta$ Lup & B2.5 IV & 3.40 & -0.24 & -3.49 & 0.00 & $\begin{array}{c}(-0.24) \\
-0.22\end{array}$ & -3.49 \\
\hline 2282 & $\zeta \mathrm{CMa}$ & B2.5 IV & 3.02 & -0.13 & -2.91 & 0.40 & -0.22 & -3.01 \\
\hline 5812 & $\tau \mathrm{Lib}$ & B2.5 V & 3.65 & -0.17 & -2.95 & 0.22 & -0.22 & -3.17 \\
\hline
\end{tabular}


Table I (Continued)

\begin{tabular}{|c|c|c|c|c|c|c|c|c|}
\hline HR No. & Star & Sp. Type & $V$ & $(B-V)$ & $1700-V$ & $E_{1700}$ & $(B-V)_{0}$ & $(1700-V)_{0}$ \\
\hline 7623 & $\theta^{1} \mathrm{Sgr}$ & B2.5 IV & 1.24 & -0.15 & -2.81 & 0.31 & -0.22 & -3.12 \\
\hline 2106 & $\gamma \mathrm{Col}$ & B2.5 IV & 4.35 & -0.21 & -2.66 & 0.04 & -0.22 & -2.70 \\
\hline 1497 & $\tau$ Tau & B3 V & 4.29 & -0.14 & -2.62 & 0.26 & -0.20 & -2.88 \\
\hline 1641 & $\eta$ Aur & B3 V & 3.19 & -0.18 & -2.83 & 0.09 & -0.20 & -2.92 \\
\hline 5191 & $\eta \mathrm{UMa}$ & B3 V & 1.86 & -0.18 & -2.76 & 0.09 & -0.20 & -2.85 \\
\hline 5626 & $\lambda$ Lup & B3 V & 4.04 & -0.20 & -2.83 & 0.00 & -0.20 & -2.83 \\
\hline 2159 & $v$ Ori & B3 IV & 4.42 & -0.15 & -2.80 & 0.22 & -0.20 & -3.02 \\
\hline 2199 & $\xi$ Ori & B3 IV & 4.48 & -0.17 & -2.86 & 0.13 & -0.20 & -2.99 \\
\hline 1934 & $\omega$ Ori & B3 $\mathrm{III}_{\mathrm{e}}$ & 4.59 & -0.11 & -2.66 & 0.40 & -0.20 & -3.06 \\
\hline 542 & $\varepsilon$ Cas & B3 $V_{p}$ & 3.38 & -0.15 & -2.53 & 0.22 & -0.20 & -2.75 \\
\hline 472 & $\alpha$ Eri & B3 $V_{p}$ & 0.49 & -0.17 & -2.60 & 0.13 & -0.20 & -2.73 \\
\hline 5712 & $\phi^{2}$ Lup & B4 V & 4.53 & -0.16 & -2.81 & 0.02 & -0.18 & -2.90 \\
\hline 226 & $v$ And & B5 V & 4.52 & -0.15 & -2.38 & 0.04 & -0.16 & -2.42 \\
\hline 1122 & $\delta$ Per & B5 III & 3.03 & -0.12 & -2.45 & 0.18 & -0.16 & -2.63 \\
\hline 8773 & $\beta$ Psc & B6 $V_{e}$ & 4.52 & -0.12 & -2.34 & 0.09 & -0.14 & -2.43 \\
\hline 8425 & $\alpha$ Gru & B7 IV & 1.73 & -0.17 & -2.18 & 0.00 & $\begin{array}{c}(-0.17) \\
-0.12\end{array}$ & -2.18 \\
\hline 1791 & $\beta$ Tau & B7 III & 1.66 & -0.13 & -2.02 & 0.00 & $\begin{array}{c}(-0.13) \\
-0.12\end{array}$ & -2.02 \\
\hline 7039 & $\phi \mathrm{Sgr}$ & B8 III & 3.17 & -0.11 & -1.72 & 0.00 & $\begin{array}{c}(-0.11) \\
-0.09\end{array}$ & -1.72 \\
\hline 1713 & $\beta$ Ori & B8 Ia & 0.15 & -0.03 & -1.33 & 0.00 & $\begin{array}{c}(-0.03) \\
-0.02\end{array}$ & -1.33 \\
\hline 2095 & $\theta$ Aur & B9.5 si & 2.63 & -0.08 & +0.01 & 0.00 & $\begin{array}{c}(-0.08) \\
-0.03\end{array}$ & +0.01 \\
\hline 7011 & $\alpha$ Lyr & $\mathrm{A} 0 \mathrm{~V}$ & 0.00 & 00.00 & -0.66 & 0.00 & 0.00 & -0.66 \\
\hline 4905 & $\varepsilon \mathrm{UMa}$ & $A 0 p$ & 1.78 & -0.03 & -0.56 & 0.00 & $\begin{array}{c}(-0.03) \\
0.00\end{array}$ & -0.56 \\
\hline 2491 & $\alpha \mathrm{CMa}$ & $A 1 V_{p}$ & 1.45 & -0.01 & -0.62 & 0.00 & $\begin{array}{c}(-0.01) \\
+0.03\end{array}$ & -0.62 \\
\hline 2088 & $\beta$ Aur & A2 V & 1.90 & +0.03 & +0.27 & 0.00 & $\begin{array}{c}(+0.03) \\
+0.06\end{array}$ & +0.27 \\
\hline $\begin{array}{l}1666 \\
1702\end{array}$ & $\beta$ Eri & A3 III & 2.78 & +0.12 & +0.20 & 0.02 & +0.10 & +0.11 \\
\hline 1702 & $\mu$ Lep & A5 $p$ & 3.29 & -0.11 & -1.85 & 0.00 & $-0.11)$ & -1.85 \\
\hline
\end{tabular}

In the process of determining effective temperatures, however, I was impressed by the sensitivity of the determinations to line blanketing and for the hottest stars to the effects of extended atmospheres. Figure 7 compares two of Cassinelli's (1971) model atmospheres with different curvatures with a $50000 \mathrm{~K}$ ATLAS model. Two effects should be noted. First, the decrease in the flux gradient in the Paschen and Balmer continua with increasing extension of the atmosphere which would therefore yield an artificially low effective temperature as has been pointed out by Heap (1972) and Conti (1972). Second, the absolute luminosity in the Lyman continuum is increased, providing more photons for ionization of interstellar clouds. In the case of extended atmospheres it is necessary to give up the concept of effective temperature and replace it by the total luminosity.

I believe that no essential improvement in the effective temperature scale for early 


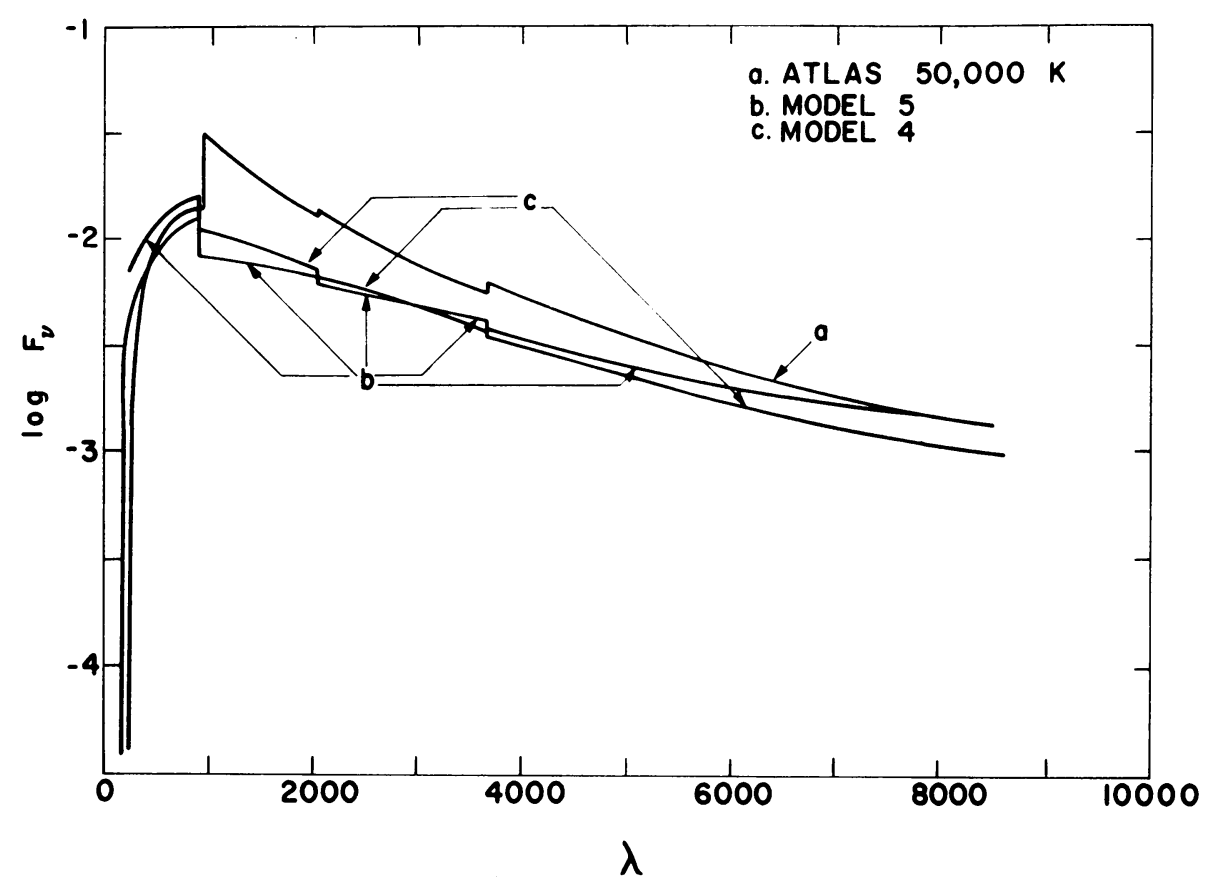

Fig. 7. Comparison of Casinelli's model atmospheres with 50000K ATLAS model.

type stars can be achieved from measurements of stellar fluxes until such time as accurate fluxes are available in the ultraviolet down to the Lyman jump. It will then be possible to determine the integrated fluxes of early type stars and hence empirical effective temperatures for those stars for which angular diameters are available and empirical bolometric corrections for others. For the very hottest stars where uncertainties in the contribution of the Lyman continuum to the total flux is important, we require further studies of NLTE and extended atmospheres.

Doherty (1972) has investigated the ultraviolet energy distribution of late type stars. The agreement with model atmosphere calculations by Gingrich is remarkably good considering the large uncertainty in blanketing corrections required for these stars. High resolution studies will be required to derive satisfactory line identifications and blanketing coefficients.

\section{Conclusion}

The basic problems requiring further investigation that have been highlighted in this discussion are primarily observational.

The resolution of the remaining discrepancies in energy determinations must be resolved. Of particular importance is a redetermination of the Balmer jump of Vega or the slope and absolute flux in the Balmer continuum. The differences in the Brackett continuum also deserve investigation.

It is necessary to resolve the difference in calibration in the ultraviolet between 
$1300 \AA$ and $3000 \AA$ Shortward of $1300 \AA$ absolute energy measures are basically nonexistent.

Line blanketing presents many important problems requiring high resolution spectroscopy particularly in the ultraviolet along with laboratory work.

Finally, theoretical work must be continued on the effects of blanketing and on extended atmospheres.

\section{References}

Arvesen, J. C., Griffin, R. N., and Pearson, B. D.: 1969, Appl. Opt. 8, 2215.

Bahner, K.: 1963, Astrophys. J. 138, 1314.

Bless, R. C. and Code, A. D.: 1972, Ann. Rev. Astron. Astrophys. 10, 197.

Bless, R. C., Fairchild, T., and Code, A. D.: 1972, NASA SP-310, 361.

Bless, R. C. and Savage, B. D.: 1972, Astrophys. J. 171, 293.

Broadfoot, A. L.: 1972, Astrophys. J. 173, 681.

Carruthers, G. R.: 1969, Astrophys. J. Letters 156, 97.

Cassinelli, J. P.: 1971, Astrophys. J. Letters 8, 105.

Code, A. D.: 1960, Stars and Stellar Systems 6, 87.

Doherty, L.: 1972, Astrophys. J., in press.

Duncan, C. H.: 1969, 'Radiation Scales and the Solar Constant', Goddard Space Flight Center Report No. X-713-69-382, Greenbelt, Md.

Evans, D. C.: 1972, NASA SP-310, 347.

Fernie, J. D., Hagen, J. P., Hagen, G. L., and McClure, L.: 1971, Publ. Astron. Soc. Pacific 83, 79.

Furukawa, P. M., Haagenson, G. L., and Scharberg, M. J.: 1967, NCAR Technical Note, No. 26.

Gaide, A. E.: 1971, in F. Labuhn and R. Lüst (eds.), 'New Techniques in Space Astronomy', IAU Symp. 41, 386.

Glushneve, I. N.: 1964, Soviet Astron. AJ 8, 163.

Hayes, D. S.: 1967, unpublished dissertation, University of California, Los Angeles.

Hayes, D. S.: 1970, Astrophys. J. 159, 165.

Heap, S. R.: 1972, Astrophys. J. Letters 10, 49.

Johnson, F. S.: 1954, Meteorol. J. 11, 431.

Johnson, H. L.: 1955, Ann. Astrophys. 18, 292.

Johnson, H. L.: 1965, Comm. Lunar Planetary Lab. 3, No. 53, 67.

Kharitonov, A. W.: 1963, Soviet Astron. AJ 7, 258.

Labs, D. and Neckel, H.: 1968, $Z$. Astrophys. 69, 1.

Martinov, D. J.: 1959, Soviet Astron. AJ 3, 633.

Matthews, T. A. and Sandage, A. R.: 1963, Astrophys. J. 138, 30.

McWhirter, R. W. P.: 1971, F. Labuhn and R. Lüst (eds.), 'New Techniques in Space Astronomy', IAU Symp. 41, 369.

Navach, C.: 1972, private communication.

Nicolet, M.: 1951, Ann. Astrophys. 14, 249.

Oke, J. B.: 1964, Astrophys. J. 140, 689.

Oke, J. B. and Schild, R. E.: 1970, Astrophys. J. 161, 1015.

Saidy, F.: 1960, Monthly Notices Roy. Astron. Soc. 115, 493.

Schild, R. E., Peterson, D. M., and Oke, J. B.: 1971, Astrophys. J. 166, 95.

Stebbins, J. and Kron, G. E.: 1957, Astrophys. J. 126, 266.

Stuart, F. E.: 1969, Astrophys. J. 157, 1255.

Tousey, R.: 1963, Space Sci. Rev. $2,3$.

Wallace, L., Caldwell, J. J., and Savage, B. D.: 1972, Astrophys. J. 172, 755.

Willstrop, R. V.: 1965, Mem. Roy. Astron. Soc. 69, 83.

\section{DISCUSSION}

Pecker: I think that you cannot rule out the measured UV energy spectra on the basis of comparison with model atmospheres, as suggested by Underhill (I quote you!), as the models are extremely un- 
certain, and their spectra still more uncertain in the UV! A small change in their parameters can change indeed many things.

Hack: In your first slide you have shown the difference between the mean of $6 \mathrm{G} 2 \mathrm{~V}$ stars and the Sun There is a sharp difference of $\sim+0.2 m$ at $\lambda^{-1} \sim 2.4$ What is the reason for that difference? Code: We don't know.

Kodaira: Even in the absolute calibration of the solar flux, we have difficulties for $\alpha<2000 \AA$. There has been a discrepancy of a factor of 3 between the values obtained by NRL (July 1966, August 1970) and those by HCO (September 1968). A Japanese group has started to make independent measurements and has obtained the first successful data with a rocket (September 1971). Our data at $\lambda=1629,1684,1739 \AA(\Delta \lambda=8.3 \AA)$ are in very close agreement with HCO data (that is, lower values). The full results will be soon published by $\mathrm{K}$. Nishi (Tokyo Observatory).

Van den Bergh: I should like to sound a very minor cautionary note! Although the agreement between the Sun and typical G2 V stars is very gratifying there is, 1 believe, one small but rather well established discrepancy. The ultraviolet cyanogen absorption in the Sun is too strong The observed solar $\mathrm{CN}$ strength corresponds to that in typical metal-rich main sequence stars with $B-V$ in the range 0.66 to 0.68 . 